MRS Advances (C) 2017 Materials Research Society

DOI: 10.1557/adv.2017.69

\title{
Synthesis and Characterization of Core-Shell Magnetic Mesoporous Silica and Organosilica Nanostructures
}

\author{
Nikola Z. Knezevic, ${ }^{1}$ Chiara Mauriello Jimenez, ${ }^{2}$ Martin Albino, ${ }^{3}$ Aleksandar Vukadinovic, ${ }^{4}$ \\ Ana Mrakovic, ${ }^{4}$ Erzsebet Illes, ${ }^{4}$ Djordje Janackovic, ${ }^{1}$ Jean-Olivier Durand, ${ }^{2}$ Claudio \\ Sangregorio ${ }^{5}$ and Davide Peddis ${ }^{4}$ \\ ${ }^{1}$ Faculty of Technology and Metallurgy, University of Belgrade, Karnegijeva 4, 11000 Belgrade, Serbia \\ 2 Institut Charles Gerhardt Montpellier, UMR 5253, CC 1701 Equipe Ingenierie \\ Moleculaire et Nano-objets, Place Eugene Bataillon, 34095 Montpellier Cedex 05, France \\ 3 INSTM and Dept. of Chemistry, Univ. of Florence, 50019, Sesto Fiorentino, Italy \\ 4 Vinca Institute of Nuclear Sciences, University of Belgrade, POB 522, 11001 Belgrade, Serbia \\ 5INSTM and CNR-ICCOM, 50019 Sesto Fiorentino, Italy \\ ${ }^{*}$ Corresponding author: Dr Nikola Z. Knezevic: nknezevic@tmf.bg.ac.rs
}

\begin{abstract}
Initial results en route toward construction of complex magnetic core-shell silica and organosilica nanotheranostics are presented. Magnetite nanoparticles are synthesized by three different methods and embedded within mesoporous silica and organosilica frameworks by different surfactant-templated procedures to produce three types of core-shell nanoparticles. Magnetite nanoparticles (15 nm in diameter) are embedded within mesoporous silica nanoparticles to produce cell-like material with predominantly one magnetite nuclei-resembling core per nanoparticle, with final particle diameter of ca. $150 \mathrm{~nm}$, specific surface area of 573 $\mathrm{m}^{2} / \mathrm{g}$ and hexagonally structured tubular pores (2.6 nm predominant diameter), extended throughout the volume of nanoparticles. Two forms of spherical core-shell nanoparticles composed of magnetite cores embedded within mesoporous organosilica shells are also obtained by employing ethylene and ethane bridged organobisalkoxysilane precursors. The obtained nanomaterials are characterized by high surface area $\left(978\right.$ and $820 \mathrm{~m}^{2} / \mathrm{g}$ ), tubular pore morphology ( 2 and $2.8 \mathrm{~nm}$ predominant pore diameters), different diameters (386 and 100-200 $\mathrm{nm}$ ), in case of ethylene- and ethane-composed organosilica shells, respectively. Different degree of agglomeration of magnetite nanoparticles was also observed in the obtained materials, and in the case of utilization of surfactant-pre-stabilized magnetite nanoparticles for the syntheses, their uniform and non-agglomerated distribution within the shells was noted.
\end{abstract}

\section{INTRODUCTION}

Intuitively, the use of inorganic materials in biological systems appears incompatible, though their effective applicability in drug delivery and imaging of biological tissues has been demonstrated by various research studies.[1] In particular, silicon-based materials are being showcased as the new generation of bioapplicable materials. [2-4] Recent research advances led to a development of nanoparticles which are even entirely made of silicon atoms, the porous silicon nanoparticles (pSiNPs) consisting of $\mathrm{Si}-\mathrm{Si}$ framework, while the surface contains $\mathrm{Si}-\mathrm{H}$ and $\mathrm{Si}-\mathrm{OH}$ moieties. This material is actually the newest member of Si-based nanoparticles which show highly promising attributes for biomedical applications, as the first synthesis and its 
in vivo biomedical application was demonstrated by M. J. Sailor group in 2009.[5] The capability of this material to spontaneously degrade in aqueous environment, due to hydrolysis of the silicon framework into silicic acid, is a very important biocompatibility feature. pSiNP is typically constructed through etching of silicon wafers, and the material is distinguished by the capacity for surface functionalization through two possible methodologies: hydrosilylation and condensation reaction with organoalkoxysilanes, which opens possibilities for construction of complex nanosystems for the effective treatment and imaging of cancer.[6-7]

Mesoporous silica nanoparticles (MSNs) have been studied since the beginning of the XXI century as promising scaffolds for construction of biomaterials, [8-9] due to high surface area, ease of surface modifications and particle diameter (100-200 nm) enabling endocytosis by diseased cells. The latter aspect allows treatment targeting, especially in the case of tumors through the enhanced permeability and retention (EPR) effect.[10] On the contrary to pSiNPs, MSN material is synthesized by bottom-up approach, from alkoxysilane precursors in basic aqueous environment, in the presence of pore-templating surfactant micelles. This methodology leads to Si-O-Si bulk composition of MSN with Si-OH moieties on their surface. Various other precursors (e.g. magnetic nanoparticles, quantum dots, organosilanes) can be introduced during the syntheses of nanomaterials or as a post-synthetic modification strategy, which allows construction of complex nanotherapeutic systems for targeting and controlling their activity.[1113]

Periodic mesoporous organosilica (PMO) nanoparticles are more recently developed Si-based material, which exhibits very promising characteristics for biomedical applications.[4, 14] The nanoparticles are synthesized from bridged organoalkoxysilanes $\left(\mathrm{R}^{\prime} \mathrm{O}_{3} \mathrm{Si}-\mathrm{R}-\mathrm{SiO}_{3} \mathrm{R}^{\prime} ; \mathrm{R}^{\prime}=\mathrm{Me}\right.$, $\mathrm{Et} ; \mathrm{R}=$ organic bridge), and the surfactant-templated synthesis conditions afford porous nature of the material. The presence of organic linker between the alkoxysilane moieties in the synthesis precursors leads to the material which contains up to $80 \%$ of the organic phase, which ultimately tailors the properties of the PMO nanoparticles. Thus, the PMO NPs could be advantageous over MSN as these materials tend to have higher surface area and more hydrophobic pore-interior, which is beneficial for increasing the loading capacities of hydrophobic drugs. In addition, the organic framework of the nanoparticles can convey novel properties to the material, e.g. photoluminescence for imaging, decreased hemolytic activity and enhanced biodegradability over their MSN counterparts.

Conferring magnetic features to Si-based nanoparticles is beneficial in terms of possibilities for achieving magnetic targeting of the diseased tissues and simultaneous MRI imaging.[15-18] In addition, alternating magnetic field can be applied to induce heating of the magnetic nanoparticles and ultimately achieve complement thermal treatment of the desired tissues.[19] In this article we present our initial results on the synthesis and characterization of novel magnetite@MSN and magnetite@PMO nanoparticles, which are intended for application in the treatment of cancer tissues through loading and controlled delivery of anticancer drugs.

\section{EXPERIMENT}

\section{Syntheses of magnetite nanoparticles}

(Mag1) by thermal decomposition: (Tris(acetylacetonato) iron(III) (Fe(acac)3) 2 mmol, Oleic acid $10 \mathrm{mmol}$ and Oleylamine $10 \mathrm{mmol}$ ) were dissolved in $25 \mathrm{~mL}$ of dibenzyl ether in a three neck flask. Nitrogen was first purged for 15 minutes and then the reaction mixture was stirred at $7000 \mathrm{rpm}$ with magnetic stirrer and heated first to $200{ }^{\circ} \mathrm{C}$ (ramp in 15 minutes), then the 
temperature was held at $200{ }^{\circ} \mathrm{C}$ for 90 minutes, raised again in 10 minutes to $300^{\circ} \mathrm{C}$ and held at $300^{\circ} \mathrm{C}$ for $1 \mathrm{~h}$, all in nitrogen atmosphere. After this time the reaction was quenched by rapid cooling to room temperature and then NPs were precipitated with ethanol $(250 \mathrm{~mL})$. The magnetite NPs were washed with ethanol twice and redispersed in chloroform.

(Mag2) magnetite nanoparticles were prepared as previously reported[20] by co-precipitation of $\mathrm{Fe}$ (III) and $\mathrm{Fe}$ (II) chlorides in aqueous environment with ammonia at $\mathrm{pH} 9$.

(Mag3) magnetite nanoparticles were synthesized according to the previously published procedure by thermal treatment of hydrated iron oxide in the presence of oleic acid in docosane.[21]

\section{Synthesis of core-shell magnetite@mesoporous silica nanoparticles (Mag1@MSN)}

Iron oxide nanocrystals (Mag1) dissolved in chloroform $(50 \mathrm{mg}$ ) were added to a solution obtained dissolving $250 \mathrm{mg}$ cetyl trimethylammonium bromide (CTAB, Aldrich, 95\%) in $20 \mathrm{~mL}$ of water. The mixture was sonicated for 30 minutes and the chloroform was boiled off from the solution with rapid stirring at $60{ }^{\circ} \mathrm{C}$. During this process the reaction mixture changed from milky white to clear solution of magnetite in aqueous CTAB stabilized solution.

Then a warm solution containing $875 \mu \mathrm{L}$ of $2.0 \mathrm{M} \mathrm{NaOH}$ in $100 \mathrm{~mL}$ miliQ water was added to the suspension of magnetite and the reaction solution was heated to $80{ }^{\circ} \mathrm{C}$ (ramp 30 minutes) and $1.25 \mathrm{~mL}$ of tetraethyl orthosilicate (TEOS, Aldrich, 98\%) was added dropwise. After two hours of rapid stirring at $80{ }^{\circ} \mathrm{C}$, the silica coated magnetite nanoparticles were collected by filtration and washed twice with water and ethanol. Yield: $320 \mathrm{mg}$ of the material.

Synthesis of core-shell magnetite@ethylene-based PMO nanoparticles (Mag2@ETY-PMO) $500 \mathrm{mg}$ of CTAB was dissolved in $120 \mathrm{~mL}$ of deionized water and $1.75 \mathrm{~mL}$ of $2 \mathrm{M} \mathrm{NaOH}$ was added. The solution was stirred at $25{ }^{\circ} \mathrm{C}$ until CTAB dissolved and $75 \mathrm{mg}$ of magnetite nanoparticles was added. Suspension was sonicated for $16 \mathrm{~h}$, heated to $80{ }^{\circ} \mathrm{C}$ and $0.25 \mathrm{~mL}$ of (3mercaptopropyl)trimethoxysilane was added, followed by $2 \mathrm{ml}$ of 2-bis(triethoxysilyl)ethylene. The solution was stirred with a mechanical stirrer $(400 \mathrm{rpm})$ at $80 \mathrm{deg}$ for $2 \mathrm{~h}$, cooled and centrifuged. The material was washed two times with ethanol. CTAB was removed from the material by sonication for $25 \mathrm{~min}$ in a solution of ammonium nitrate in ethanol $(6 \mathrm{~g} / \mathrm{L})$ twice and subsequent centrifugation. The material was washed with ethanol twice, once with acetone and dried under high vacuum at $80^{\circ} \mathrm{C}$ for $2 \mathrm{~h}$.

\section{Synthesis of core-shell magnetite@ethane-based PMO nanoparticles (Mag3@ETA-PMO)}

A mixture of CTAB $(250 \mathrm{mg})$, distilled water $(120 \mathrm{~mL})$, and sodium hydroxide $(875 \mu \mathrm{L}, 2 \mathrm{M}$ aqueous solution) was stirred at $80{ }^{\circ} \mathrm{C}$ for 50 minutes at $750 \mathrm{rpm}$ in a $250 \mathrm{~mL}$ three-neck round bottom flask. At the same time, a mixture of a solution of $\mathrm{Fe}_{3} \mathrm{O}_{4}$ particles $(1 \mathrm{~mL}$ of particles in 1 $\mathrm{mL}$ of water) was sonicated at $50{ }^{\circ} \mathrm{C}$ for 50 minutes. Then, the solution of $\mathrm{Fe}_{3} \mathrm{O}_{4}$ particles was added to the surfactant solution, and it was stirred at $80{ }^{\circ} \mathrm{C}$ for $1 \mathrm{~h}$. After that, 1,2bis(triethoxysilyl) ethane $(660 \mu \mathrm{L})$ was added and the condensation reaction was conducted at $80^{\circ} \mathrm{C}$ for $2 \mathrm{~h}$. Afterwards, the solution was cooled to room temperature while stirring; fractions were gathered in propylene tubes and collected by centrifugation for 20 minutes at $20 \mathrm{Krpm}$. The samples were then washed three times with a solution of $\mathrm{NH}_{4} \mathrm{NO}_{3}$ in $\mathrm{EtOH}\left(6 \mathrm{~g} \mathrm{~L}^{-1}\right)$, water, and ethanol. Each washing was followed by centrifugation collection of the sample in the same manner. The as-prepared material was dried under vacuum for a few hours. $130 \mathrm{mg}$ of the product were obtained. 


\section{RESULTS AND DISCUSSION}

Synthesis of magnetite nanoparticles by thermal decomposition of tris(acetylacetonato) iron(III) $\left(\mathrm{Fe}(\mathrm{acac})_{3}\right)$ complex was performed in a high boiling solvent (dibenzyl ether). The characteristics of the resulting synthesized magnetite nanoparticles can be seen on XRD and TEM images on Figure 1. Analysis of XRD patterns revealed that the nanoparticles have magnetite/maghemite structure and that the crystallite size is $15 \mathrm{~nm}$. TEM images show that the sample is monodisperse and that the individual nanoparticles are well resolved and not agglomerated, which can be attributed to the presence of oleic acid and oleylamine on their surface.

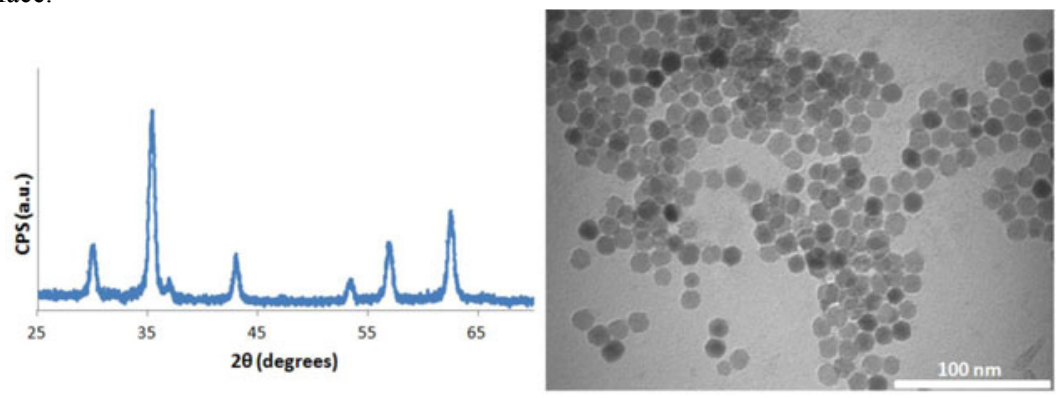

Figure 1. XRD pattern and TEM image of magnetic nanoparticles (I)

These magnetic nanoparticles (NPs) were subsequently used for construction of core-shell nanoparticles, with mesoporous silica as the shell in the material. Hydrophobic oleate-capped magnetic NPs were transferred into aqueous environment by addition of cetyltrimethylammonium bromide, which yielded a clear aqueous suspension of magnetic NPs after removal of chloroform by heating at $60{ }^{\circ} \mathrm{C}$. The hydrolysis and condensation of silica precursors in basic environment at $80{ }^{\circ} \mathrm{C}$ was then employed for formation of mesoporous silica nanoparticles with the embedded magnetic nanoparticles as the cores (Mag1@MSN). As can be observed on TEM images of the prepared core-shell material (Figure 2), the cell-like core-shell nanoparticles were successfully formed with a diameter of ca. $150 \mathrm{~nm}$, where each nanoparticle (cell) contains embedded well-separated one or more magnetic NPs (nuclei). The presence of mesopores within the silica nanoshell is evidenced on higher magnification TEM images (Fig 3). 


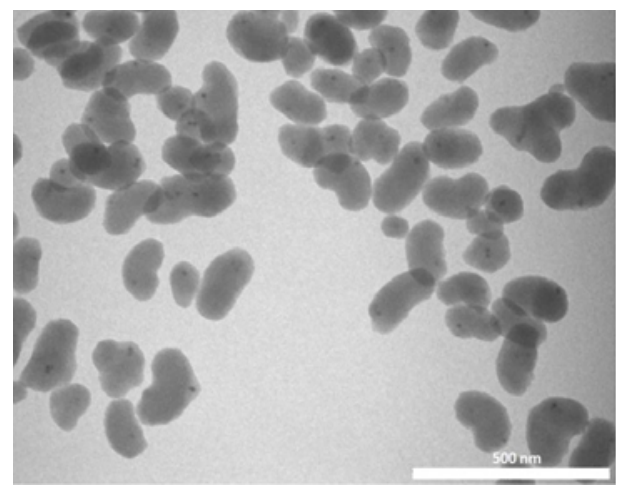

Figure 2. Low magnification TEM of core-shell magnetic MSN material showing mesoporous silica nanoparticles with embedded magnetic nanoparticles (black dots).

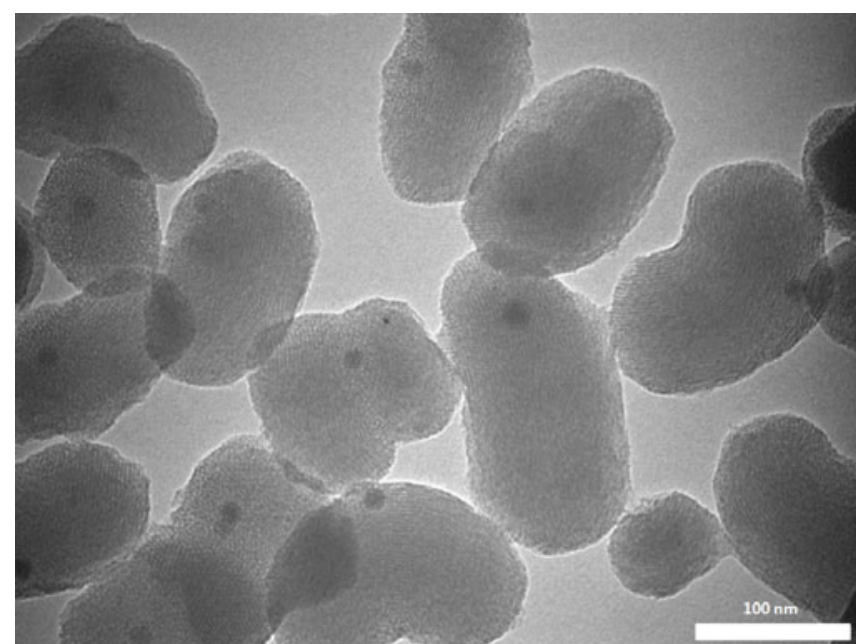

Figure 3. High magnification TEM image of core-shell Mag1@MSN showing the presence of mesopores within the silica nanoparticles (brighter lines and dots)

Nitrogen sorption measurements revealed that the material exhibit Brunauer-Emmett-Teller (BET) specific surface area of $573 \mathrm{~m}^{2} / \mathrm{g}$ and predominant Barrett-Joyner-Halenda (BJH) pore diameter of $2.6 \mathrm{~nm}$. XRD measurements at low angle showed that the Mag1@MSN material contains hexagonally packed mesopores as evident on Figure 4a. High angle XRD evidences the presence of magnetite/maghemite pattern within the Mag1@MSN material (Figure 4b). 

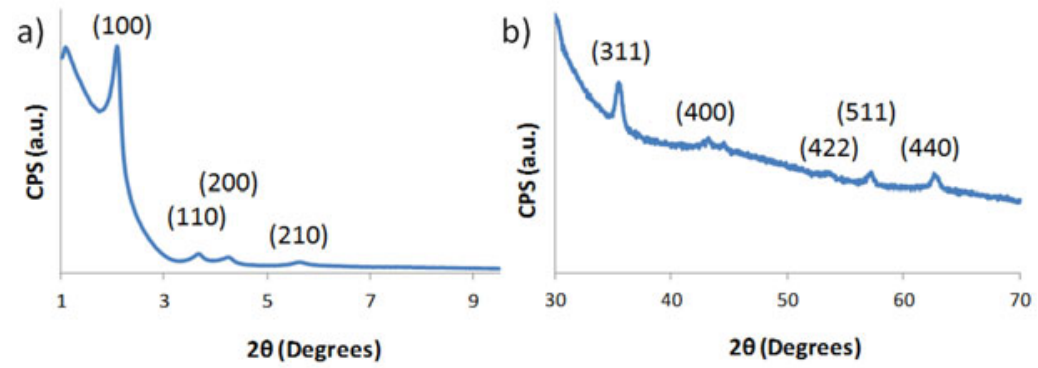

Figure 4. XRD measurement data for Mag1@MSN at a) low angle and b) high angle

Synthesis of Mag2@ETY-PMO core-shell magnetic nanostructures was performed by a methodology analogous to the one employed for Mag1@MSN. However, instead of TEOS, as a silica precursor bis(triethoxysilyl)ethylene was employed, with addition of small amount of (3mercaptopropyl)trimethoxysilane, which served as an initial silica layer on the surface of magnetic nanoparticles. TEM analysis (Figure 5) revealed the formation of spherical core-shell PMO nanoparticles with average diameter of $386 \mathrm{~nm}$, as determined by DLS analysis.
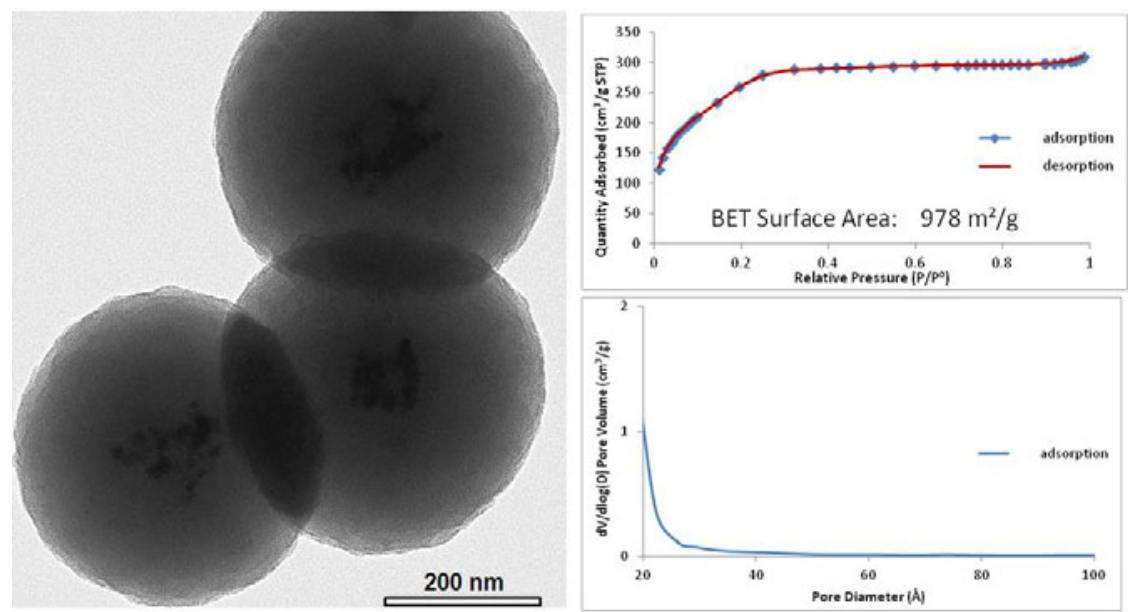

Figure 5. Characterization of Mag2@ETY-PMO by TEM (left panel) and nitrogen sorption measurement (right panel, BET (top) and BJH (bottom)).

On the contrary to the Mag1@MSN material, magnetic nanoparticles in the Mag2@ETY-PMO material are not well resolved and seem agglomerated within the core of PMO nanoparticles. This result is expected since the initial magnetite nanoparticles used as precursors were not surface modified and hence their complete separation from each other could not be achieved, even though a prolonged sonication period $(16 \mathrm{~h})$ was employed. Nitrogen sorption 
measurements revealed that the material exhibits high BET surface area $\left(978 \mathrm{~m}^{2} / \mathrm{g}\right)$ and that the predominant $\mathrm{BJH}$ pore diameter is below $2 \mathrm{~nm}$.

In the case of Mag3@ETA-PMO material, TEM analysis reveals that the magnetite nanoparticles are better dispersed within the PMO framework (Figure 6), which is again due to the presence of surface-stabilizing oleic acid layer on Mag3 nanoparticles.

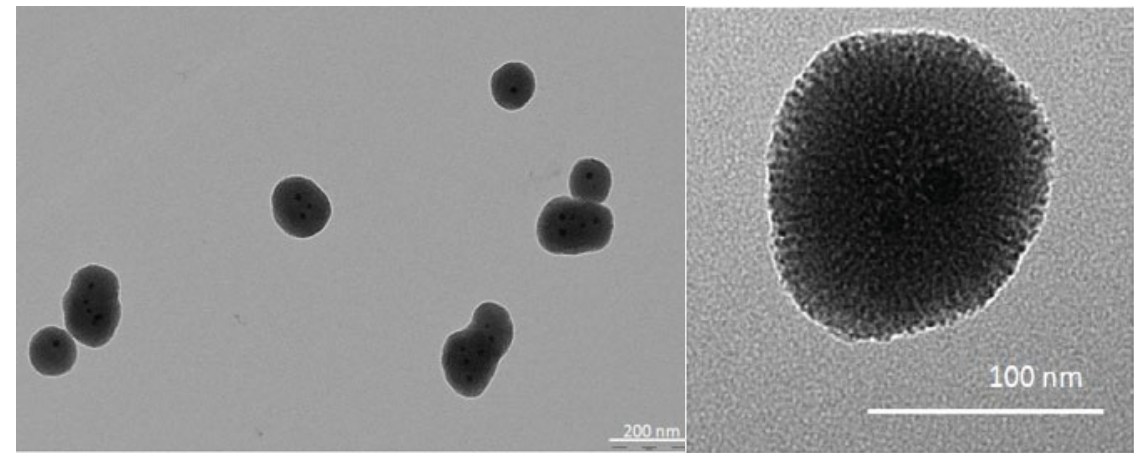

Figure 6. Characterization of Mag3@ETA-PMO by TEM

The mesoporous structure of Mag3@ETA-PMO was confirmed by nitrogen sorption analysis (Figure 7). Specific surface area of $820 \mathrm{~m}^{2} / \mathrm{g}$ (BET) was obtained while the BJH pore size analysis revealed the predominance of $2.8 \mathrm{~nm}$ wide mesopores.
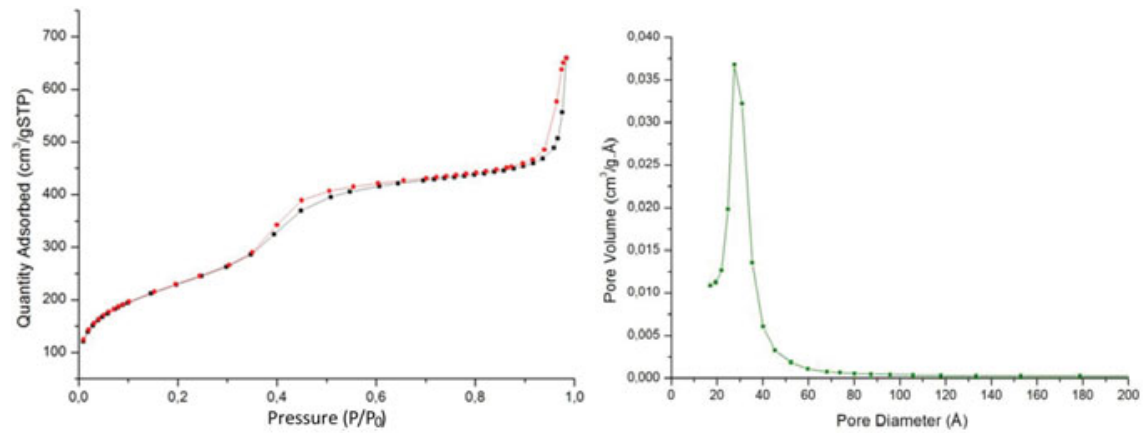

Figure 7. Nitrogen sorption characterization of Mag3@ETA-PMO, by BET (left panel) and BJH (right panel).

\section{CONCLUSIONS}

We successfully synthesized three novel magnetic core-shell nanomaterials containing magnetite cores within mesoporous silica, ethylene bridged or ethane bridged periodic organosilica organosilica nanoparticles. The materials are characterized by transmission electron 
microscopy, XRD and nitrogen sorption measurements and their characteristics (small diameter, uniform morphology, high porosity, and high surface area) are very promising for future applications in construction of nanosystems for simultaneous MRI diagnostics and therapy. In case of core-shell nanoparticles containing mesoporous silica shells, predominantly one magnetite nanoparticle of $15 \mathrm{~nm}$ in diameter per final core-shell nanoparticle (ca. $150 \mathrm{~nm}$ in diameter) is obtained. The final material exhibited specific surface area of $573 \mathrm{~m}^{2} / \mathrm{g}$ and hexagonally structured tubular pores $(2.6 \mathrm{~nm}$ predominant diameter), extended throughout the volume of nanoparticles. Core-shell, magnetite embedded mesoporous organosilica nanoparticles with high surface area (978 and $820 \mathrm{~m}^{2} / \mathrm{g}$ ), tubular pore morphology ( 2 and $2.8 \mathrm{~nm}$ predominant pore diameters), different average diameters (386 and 100-200 $\mathrm{nm}$ ), are also obtained by employing ethylene and ethane bridged organobisalkoxysilane precursors, respectively. Dispersion and stabilization of magnetic nanoparticles with surfactants before their introduction for the syntheses of core-shell nanoparticles yields core-shell nanoparticles with uniform and non-aggregated distribution of magnetite cores within silica or organosilica shells. Hence, the employment of surfactant-pre-stabilized magnetite can be regarded as preferred methodology for the synthesis of core-shell silica or organosilica nanoparticles for biomedical applications. Considering the two synthesized organosilica core-shell nanomaterials, the one synthesized with 1,2-bis(triethoxysilyl) ethane as precursor can be regarded as the better fitting for biomedical applications due to smaller particle diameter and uniform pore morphology. Further research is intended to compare the capabilities of magnetic mesoporous silica and organosilica nanoparticles for drug loading and delivery capacities in order to fully exploit the properties of these core-shell nanomaterials for applications in therapy and imaging.

\section{ACKNOWLEDGMENTS}

For financial support, N. Z. K. is thankful to CNR Short Term Mobility Program, CNRS Chaire Total Visiting Professorship Program and the Ministry of Science and Technological Development of the Republic of Serbia (Grant no. III45019). The authors from Vinca Institute and N. Z. K. acknowledge the support from MagBioVin FP7 Era Chairs project.

\section{REFERENCES}

[1] E. B. Ehlerding, F. Chen and W. Cai, Advanced Science 3, 1500223 (2016)

[2] V. Stojanovic, F. Cunin, J. O. Durand, M. Garcia and M. Gary-Bobo, Journal of Materials Chemistry B (2016)

[3] N. Z. Knezevic and J.-O. Durand, ChemPlusChem 80, 26 (2015)

[4] J. G. Croissant, X. Cattoen, M. Wong Chi Man, J.-O. Durand and N. M. Khashab, Nanoscale 7, 20318 (2015)

[5] J.-H. Park, L. Gu, G. von Maltzahn, E. Ruoslahti, S. N. Bhatia and M. J. Sailor, Nat Mater 8, 331 (2009)

[6] N. Z. Knezevic, V. Stojanovic, A. Chaix, E. Bouffard, K. E. Cheikh, A. Morere, M. Maynadier, G. Lemercier, M. Garcia, M. Gary-Bobo, J.-O. Durand and F. Cunin, Journal of Materials Chemistry B 4, 1337 (2016)

[7] A. Chaix, K. El Cheikh, E. Bouffard, M. Maynadier, D. Aggad, V. Stojanovic, N. Knezevic, M. Garcia, P. Maillard, A. Morere, M. Gary-Bobo, L. Raehm, S. Richeter, J.-O. Durand and F. Cunin, Journal of Materials Chemistry B 4, 3639 (2016) 
[8] M. Vallet-Regi, A. Rámila, R. P. del Real and J. Pérez-Pariente, Chemistry of Materials 13, $308(2001)$

[9] C.-Y. Lai, B. G. Trewyn, D. M. Jeftinija, K. Jeftinija, S. Xu, S. Jeftinija and V. S. Y. Lin, Journal of the American Chemical Society 125, 4451 (2003)

[10] J. Lu, M. Liong, Z. Li, J. I. Zink and F. Tamanoi, Small 6, 1794 (2010)

[11] N. Z. Knezevic, J. Mrdanovic, I. Borisev, S. Milenkovic, D. Janackovic, F. Cunin and A. Djordjevic, RSC Advances 6, 7061 (2016)

[12] N. Z. Knezevic, E. Ruiz-Hernandez, W. E. Hennink and M. Vallet-Regi, RSC Advances 3, $9584(2013)$

[13] W. Fan, B. Shen, W. Bu, F. Chen, Q. He, K. Zhao, S. Zhang, L. Zhou, W. Peng, Q. Xiao, D. $\mathrm{Ni}$, J. Liu and J. Shi, Biomaterials 35, 8992 (2014)

[14] C. M. Jimenez, N. Z. Knezevic, Y. G. Rubio, S. Szunerits, R. Boukherroub, F. Teodorescu, J. G. Croissant, O. Hocine, M. Seric, L. Raehm, V. Stojanovic, D. Aggad, M. Maynadier, M. Garcia, M. Gary-Bobo and J.-O. Durand, Journal of Materials Chemistry B 4, 5803 (2016) [15] C. Tudisco, M. T. Cambria, F. Sinatra, F. Bertani, A. Alba, A. E. Giuffrida, S. Saccone, E. Fantechi, C. Innocenti, C. Sangregorio, E. Dalcanale and G. G. Condorelli, Journal of Materials Chemistry B 3, 4134 (2015)

[16] B. H. McDonagh, G. Singh, S. Hak, S. Bandyopadhyay, I. L. Augestad, D. Peddis, I. Sandvig, A. Sandvig and W. R. Glomm, Small 12, 301 (2016)

[17] N. Z. Knezevic, Journal of Nanoscience and Nanotechnology 16, 4195 (2016)

[18] N. Z. Knezevic, Processing and Application of Ceramics 8, 109 (2014)

[19] E. Fantechi, C. Innocenti, M. Zanardelli, M. Fittipaldi, E. Falvo, M. Carbo, V. Shullani, L.

Di Cesare Mannelli, C. Ghelardini, A. M. Ferretti, A. Ponti, C. Sangregorio and P. Ceci, ACS nano 8, 4705 (2014)

[20] N. Z. Knezevic, I. I. Slowing and V. S. Y. Lin, Chempluschem 77, 48 (2012)

[21] S. Dib, M. Boufatit, S. Chelouaou, F. Sadi-Hassaine, J. Croissant, J. Long, L. Raehm, C.

Charnay and J. O. Durand, RSC Advances 4, 24838 (2014) 